\title{
Modificações Evolutivas da Necessidade de Cirurgia de Revascularização Miocárdica de Emergência em Indivíduos Submetidos a Intervenção Coronária Percutânea - Análise de 9.938 Pacientes
}

\author{
Carlos Augusto Homem de Magalhães Campos¹, Mila Yugar², Antonia Petrizzo², Expedito E. Ribeiro', \\ Henrique Ribeiro', Andre G. Spadaro', Marco Perin ${ }^{1}$, Pedro A. Lemos'1, Eulogio Martinez', \\ Gilberto Marchiori' ${ }^{1}$, José Antonio F. Ramires ${ }^{1}$
}

\section{RESUMO}

Introdução: As intervenções coronárias percutâneas (ICPs) em centros sem cirurgia cardíaca representam uma real opção no contexto atual da cardiologia intervencionista. O propósito deste estudo foi avaliar as mudanças na incidência e indicações para cirurgia de revascularização miocárdica de emergência em pacientes submetidos a ICP de 1992 a 2005. Método: No total, 9.938 pacientes submetidos a ICP em dois centros foram divididos em três grupos: período pré-stent, de 1992 a $1996(\mathrm{n}=2.500)$, período intermediário, de 2002 a 2003 ( $\mathrm{n}=3.711)$, e período mais recente, de 2004 a $2005(n=3.727)$. Resultados: Com o advento dos stents passou-se a abordar uma população mais idosa $(58,2 \pm 10,7$ anos vs. $62,7 \pm 11,7$ anos vs. $67,8 \pm 11,6$ anos; $\overline{\mathrm{P}}=0,01)$, com lesões mais complexas, do tipo B2/C $(52,2 \%$ vs. $72,7 \%$ vs. $79 \% ; P<0,01)$, mas com melhora nos resultados de oclusão aguda $(7,8 \%$ vs. $1,7 \%$ vs. $1,1 \%$; $\mathrm{P}<0,01)$ e de cirurgia de revascularização miocárdica de emergência $(1,2 \%$ vs. $1,1 \%$ vs. $0,7 \% ; P<0,01)$ e consequente menor mortalidade $(3,3 \%$ vs. $2,2 \%$ vs. $2 \%$; $\mathrm{P}<0,01)$. Foram identificados como preditores da necessidade de cirurgia de emergência o padrão de acometimento coronário multiarterial [odds ratio (OR) 3,42, intervalo de confiança de 95\% (IC 95\%) 1,75-6,7], infarto agudo do miocárdio (OR 3,65, IC 95\% 2,3-5,8) e complexidade das lesões [tipo B2/C, American Heart Association/American College of Cardiology - AHA/ACC (OR 3,27, IC 95\% 1,6$6,75)]$. A característica que exerceu efeito protetor foi a utilização de stent (OR 0,58, IC 95\% 0,4-0,85). Conclusão: Os avanços da técnica, do material e da farmacoterapia adjunta têm contribuído com a ICP no sentido de diminuir

\section{ABSTRACT}

Evolutionary Changes in the Need of Emergency Bypass Surgery in Individuals Undergoing Percutaneous Coronary Interventions Analysis of 9,938 Patients

Background: Percutaneous coronary interventions $(\mathrm{PCI})$ in centers without cardiac surgery represent a real option in the current context of interventional cardiology. The purpose of this study was to evaluate changes in the incidence and indications for coronary artery bypass grafting in patients undergoing PCl from 1992 to 2005. Method: Data from 9,938 patients undergoing $\mathrm{PCl}$ in two centers were divided into three groups: pre-stent period, from 1992 to 1996 ( $n=$ 2,500), intermediate period, from 2002 to 2003 ( $n=3,711$ ) and more recent period, from 2004 to 2005 ( $n=3,727$ ). Results: Since the advent of stents, an older population $(58.2 \pm 10.7$ years vs. $62.7 \pm 11.7$ years vs. $67.8+11.6$ years; $\mathrm{P}=0.01$ ), with more complex type B2/C lesions $(52.2 \%$ vs. $72.7 \%$ vs $79 \% ; \mathrm{P}<0.01)$ is being treated, with a lower rate of acute vessel occlusion $(7.8 \%$ vs. $1.7 \%$ vs. $1.1 \% ; \mathrm{P}<0.01)$, emergency coronary artery bypass grafting $(1.2 \%$ vs. $1,1 \%$ vs. $0.7 \% ; \mathrm{P}<0.01)$ and mortality $(3.3 \%$ vs. $2.2 \%$ vs. $2 \% ; \mathrm{P}<0.01)$. Predictors of the need of emergency bypass surgery were multivessel disease [odds ratio (OR) $3.42,95 \%$ confidence interval $(95 \% \mathrm{Cl}) 1.75-6.7)$, acute myocardial infarction (OR 3.65, 95\% Cl 2.3-5.8) and lesion complexity [type B2/C, American Heart Association/American College of Cardiology - AHA/ACC (OR 3.27, 95\% Cl 1.6-6.75)]. The use of stents, however, conferred a protective effect against the need of emergency bypass surgery

1 Instituto do Coração do Hospital das Clínicas da Faculdade de Medicina da Universidade de São Paulo - São Paulo, SP, Brasil. 2 Hospital e Maternidade São Luiz - São Paulo, SP, Brasil. Correspondência: Carlos Augusto Homem de Magalhães Campos. Av. Dr. Enéas Carvalho de Aguiar, 44 - São Paulo, SP, Brasil CEP 05403-900

E-mail: cm-campos@uol.com.br

Recebido em: 1\%/3/2010 • Aceito em: 11/5/2010 
a necessidade de cirurgia de revascularização miocárdica de emergência.

DESCRITORES: Revascularização miocárdica. Emergências. Stents.

A s intervenções coronárias percutâneas (ICPs) foram realizadas pela primeira vez em $1977^{1} \mathrm{e}$ desde então ganharam rápida aceitação como opção terapêutica no tratamento da doença arterial coronária. Entre 1977 e 1986, cateteres, fios-guia e balões de angioplastia foram aperfeiçoados, com melhor navegabilidade e tolerância a maiores pressões de insuflação. A segunda ferramenta de grande relevância da cardiologia intervencionista foi a introdução dos stents coronários, que tinham como objetivo conter as microdissecções e fornecer suporte mecânico, abolindo o recuo elástico e o remodelamento do vaso relacionado à reestenose. Concomitantemente, os avanços na farmacoterapia adjunta, com associação de antiagregantes plaquetários, permitiu progressivo aumento dos índices de sucesso das intervenções coronárias percutâneas. $^{2}$ Dessa forma, o perfil dos pacientes e as lesões passaram por mudanças nos últimos anos, com ampliação das indicações desse procedimento, abordando lesões e pacientes cada vez mais complexos, porém com progressivos aumentos na segurança e na eficácia da intervenção coronária percutânea. ${ }^{3-8}$ Por esses motivos, com o passar dos anos, estudos internacionais demonstraram significativa redução dos índices de cirurgia cardíaca de emergência após revascularização percutânea ${ }^{9,10}$, sendo recentemente questionada a necessidade de retaguarda de uma equipe de cirurgia cardíaca quando da realização dos procedimentos de cardiologia intervencionista. Nesse contexto, buscamos avaliar os riscos, os resultados e a ocorrência de cirurgias cardíacas de emergência de centros terciários brasileiros de grande volume, mas com retaguarda de cirurgia cardiotorácica.

\section{MÉTODO}

Este foi um estudo retrospectivo, observacional, realizado em dois hospitais-escola, referência para cardiologia intervencionista de alta complexidade. A população do estudo foi composta por todos os pacientes submetidos a intervenção coronária percutânea nesses centros. Para avaliar as mudanças na incidência e as características clínicas para indicação de cirurgia de revascularização miocárdica de emergência em pacientes submetidos a intervenção coronária percutânea, essa população foi dividida em três períodos:

- Período inicial: população composta por 2.500 pacientes tratados com angioplastia coronária com balão, entre 1992 e 1996, no Hospital Unicor (São Paulo, SP).
(OR 0.58, 95\% Cl 0.4-0.85). Conclusion: Advances in technology, devices and adjunctive pharmacotherapy have improved $\mathrm{PCl}$, reducing the need of emergency coronary artery bypass grafting.

KEY-WORDS: Myocardial revascularization. Emergencies. Stents.

- Período intermediário: 3.711 indivíduos tratados com a utilização de stents, nos anos de 2002 e 2003, no Instituto do Coração do Hospital das Clínicas da Faculdade de Medicina da USP (InCor-HCFMUSP, São Paulo, SP).

- Período mais recente: 3.727 participantes tratados com a utilização de stents, no período entre janeiro de 2004 e dezembro de 2005, no InCor (São Paulo, SP).

\section{Definições}

A coleta de dados demográficos, clínicos e do procedimento obedeceu ao preenchimento de formulários previamente estabelecidos, em banco de dados dedicado. Os eventos adversos pesquisados correspondem ao período intra-hospitalar da internação índice do procedimento percutâneo. A cirurgia de revascularização de emergência foi definida como cirurgia cardíaca no período periprocedimento, com o intuito de evitar a ocorrência de óbito ou morbidade. As cirurgias realizadas eletivamente por insucesso não foram consideradas. As indicações para cirurgia de emergência incluíram oclusão aguda do vaso, dissecção coronária extensa, revascularização incompleta, perfuração coronária, dilatação malsucedida com instabilidade clínica ou qualquer outra situação que resultasse em comprometimento hemodinâmico ou que necessitasse intervenção a céu aberto. Oclusão arterial aguda foi definida como evidência de isquemia miocárdica e redução crítica ou completa do fluxo coronário no vaso previamente dilatado, levando a nova revascularização (percutânea ou cirúrgica) ou infarto do miocárdio durante ou após a angioplastia. Sucesso do procedimento foi definido como obtenção de sucesso angiográfico e ausência de complicações clínicas maiores (morte, infarto agudo do miocárdio com supradesnivelamento do segmento ST e cirurgia de revascularização miocárdica de emergência). A morfologia das lesões foi classificada de acordo com as definições do American College of Cardiology/American Heart Association (ACC/AHA) modificadas por Ellis et al. ${ }^{4}$

\section{Características analisadas}

Esta análise buscou determinar a ocorrência, nos três períodos, de desfechos hospitalares relacionados a segurança e eficácia do tratamento percutâneo. Foram comparados, entre os grupos, índices de sucesso do procedimento, oclusão arterial aguda, cirurgia cardíaca de emergência e óbito. 


\section{Análise estatística}

As variáveis contínuas foram descritas como média \pm desvio padrão e comparadas por ANOVA. As variáveis categóricas foram representadas como sua porcentagem e comparadas com teste de qui-quadrado. Utilizando a regressão de Cox, foram empregados modelos univariados e multivariados para identificar a relação entre possíveis preditores basais e necessidade de cirurgia de emergência durante o seguimento clínico. As variáveis utilizadas nesses modelos foram: idade, sexo, diabetes, quadro clínico de admissão, intervenção coronária percutânea prévia, cirurgia de revascularização miocárdica prévia, insuficiência cardíaca, hipertensão arterial sistêmica, morfologia das lesões e utilização de stents. Todos os testes foram bicaudais e o valor de $\mathrm{P}<0,05$ foi considerado significativo.

\section{RESULTADOS}

\section{Características dos pacientes submetidos a intervenção coronária percutânea}

As características dos 9.938 pacientes submetidos a tratamento coronário percutâneo estão descritas na Tabela 1 e demonstram progressivo aumento da complexidade das intervenções coronárias percutâneas. Comparado com o período inicial, os pacientes dos períodos intermediário e mais recente eram mais idosos $(58,2+10,7$ anos vs. $62,7+11,7$ anos vs. $67,8+11,6$ anos, respectivamente; $\mathrm{P}<0,01)$, com maior participação do sexo feminino (29,5\% vs. $33,3 \%$ vs. $32,2 \%$, respectivamente; $\mathrm{P}<0,01)$, maior número de cirurgias de revascularização miocárdica prévias (13,1\% vs. 17,8\% vs. $15,3 \%$, respectivamente; $P=0,01)$ e de intervenções coronárias percutâneas prévias (13,4\% vs. 20,7\% vs. $21,4 \% ; \mathrm{P}<0,01)$. À medida que avançamos no tempo também observamos, progressivamente, abordagem de lesões mais complexas AHA/ACC tipo B2/C (52,2\% vs. $72,7 \%$ vs. $79,0 \% ; P<0,01)$.

\section{Desfechos clínicos intra-hospitalares}

Conforme demonstrado nas Figuras 1 e 2, com as novas tecnologias foi observado aumento dos índices de sucesso do procedimento, ocorrendo em 93,2\% dos pacientes na era da angioplastia convencional, em 96,6\% no período intermediário e em $97,7 \%$ no período mais recente $(P<0,01)$. Comportamento inverso foi observado com os índices de oclusão aguda, sendo de $7,8 \%$ para intervenção com balão, de $1,7 \%$ no período intermediário e de $1,1 \%$ na última fase $(P<0,01)$. Da mesma forma, houve redução progressiva dos índices de cirurgia cardíaca de emergência $(1,2 \%$ vs. $1,1 \%$ vs. $0,7 \% ; P<0,01)$. Do período do balão para a época moderna também foi observada diferença nos índices de mortalidade intra-hospitalar $(3,3 \%$ vs. $2,2 \%$ vs. $2 \%$; $P=0,01)$. Vale ressaltar que aqui estão inclusos os pacientes com infarto agudo do miocárdio e submetidos a intervenção coronária percutânea primária.

\section{Diferenças entre os grupos na era do stent}

A Tabela 2 ilustra as principais diferenças dos tratamentos entre os grupos nos períodos intermediário e mais recente. No momento do implante do stent,

TABELA 1

Características dos pacientes de acordo com o período

\begin{tabular}{|c|c|c|c|c|}
\hline & $\begin{array}{c}\text { Período } \\
\text { inicial } \\
(n=2.500)\end{array}$ & $\begin{array}{c}\text { Período } \\
\text { intermediário } \\
(n=3.711)\end{array}$ & $\begin{array}{c}\text { Período } \\
\text { mais recente } \\
(n=3.727)\end{array}$ & $\mathbf{P}$ \\
\hline Idade média, anos & $58,2 \pm 10,7$ & $62,7 \pm 11,7$ & $67,8 \pm 11,6$ & $<0,01$ \\
\hline Sexo masculino, $\%$ & 70,5 & 66,7 & 67,8 & $<0,01$ \\
\hline Diabetes, \% & 17,9 & 28,5 & 30,1 & $<0,01$ \\
\hline Hipertensão arterial, \% & 45,4 & 75,8 & 82,6 & $<0,01$ \\
\hline Insuficiência cardíaca, \% & 17,4 & 11 & 10,6 & 0,01 \\
\hline Quadro clínico,\% & & & & $<0,01$ \\
\hline Angina estável & 29,5 & 36,2 & 36,9 & \\
\hline SCA sem supra & 39,5 & 32,1 & 33,2 & \\
\hline IAM com supra & 31 & 31,7 & 29,9 & \\
\hline CRM prévia, \% & 13,1 & 17,8 & 15,3 & 0,01 \\
\hline ICP prévia, \% & 13,4 & 20,7 & 21,4 & $<0,01$ \\
\hline Padrão coronário multiarterial, \% & 40,5 & 63 & 63,8 & $<0,01$ \\
\hline Lesões tipo B2/C (AHA/ACC), \% & 52,2 & 72,7 & 79 & $<0,01$ \\
\hline
\end{tabular}




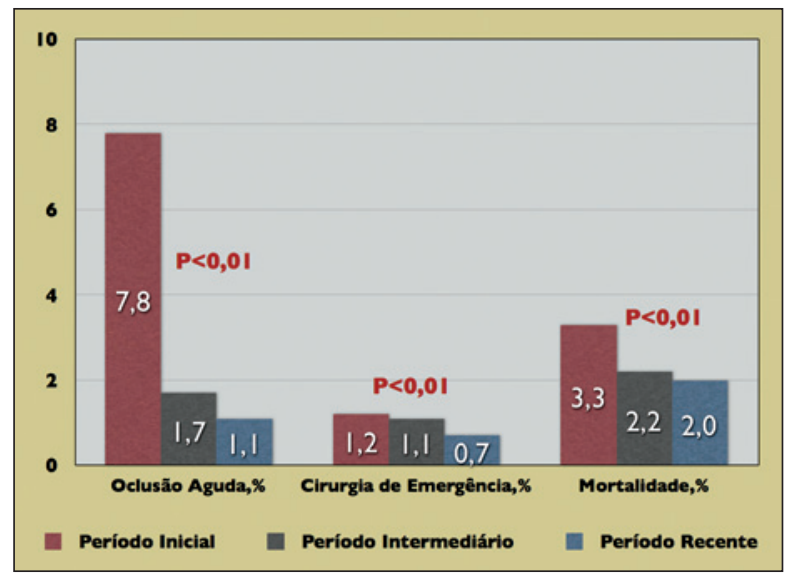

Figura 1 - Índices de sucesso do procedimento de acordo com o período da intervenção coronária percutânea.

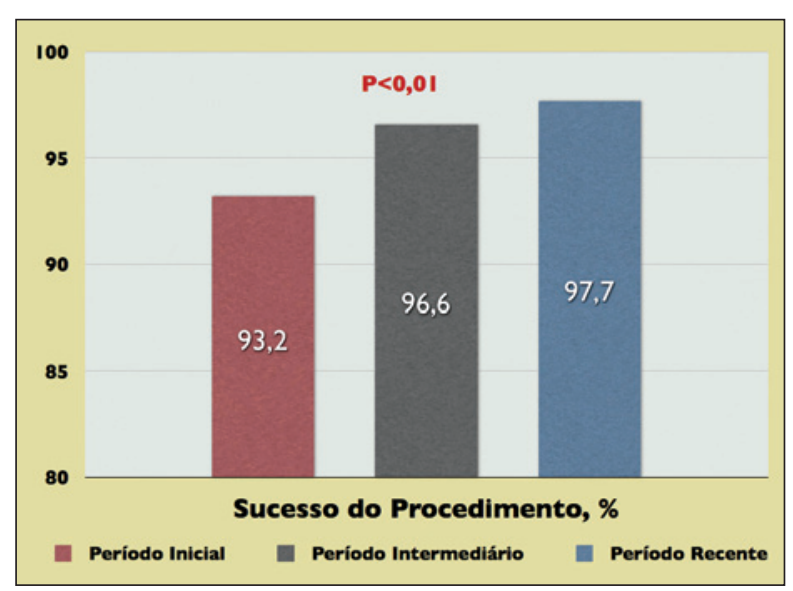

Figura 2 - Desfechos intra-hospitalares de acordo com o período da intervenção coronária percutânea.

os indivíduos tratados mais recentemente recebiam, com maior frequência, betabloqueadores (68\% vs. 63\%; $\mathrm{P}<0,01)$, inibidores da enzima conversora da angiotensina $(49,3 \%$ vs. $45,2 \% ; P<0,01)$ e estatinas $(52,5 \%$ vs. $42,8 \% ; P<0,01)$. Não houve diferenças quanto à utilização, prévia ao procedimento, de ácido acetilsalicílico, clopidogrel ou inibidores de glicoproteína Ilb/IIla. Stents foram empregados em $84,3 \%$ das lesões no período intermediário e em $88,5 \%$ das lesões no período mais recente $(P<0,01)$. Quando comparados os dois grupos de intervenção coronária percutânea com stent, apesar das lesões mais complexas B2/C tratadas no grupo mais recente $(72,7 \%$ vs. $79,1 \%$; $\mathrm{P}<0,01)$, não foi observada mudança nas taxas de oclusão aguda $(1,45 \%$ vs. $1,1 \% ; P=0,2)$, mas ainda assim houve diminuição da necessidade de cirurgia de revascularização de emergência (1,2\% vs. 0,7\%; $\mathrm{P}=0,04)$, sem, no entanto, influenciar a mortalidade global $(2,2 \%$ vs. $2 \% ; P=0,8)$.

Conforme ilustrado na Tabela 3, foram identificados como preditores independentes da necessidade de cirurgia de emergência o padrão de acometimento coronário multiarterial [odds ratio (OR) 3,42; intervalo de confiança de 95\% (IC 95\%) 1,75-6,7), a presença de infarto agudo do miocárdio (OR 3,65; IC 95\% 2,3-5,8) e a complexidade das lesões (tipo B2/C AHA/ACC [OR 3,27; IC 95\% 1,6-6,75]). A única característica que exerceu efeito protetor sobre a necessidade de intervenção cirúrgica foi a utilização de stent (OR 0,58; IC 95\% 0,4-0,85).

\section{DISCUSSÃO}

O presente estudo determinou a evolução temporal das intervenções percutâneas com a utilização dos stents e as modificações nas estratégias farmacológicas e suas correlações com a necessidade de cirurgia de emergência em dois hospitais de grande volume de intervenções coronárias percutâneas, com retaguarda de serviços de cirurgia cardiotorácica. Os principais achados deste registro foram: houve significativa mudança no perfil dos pacientes submetidos a intervenção coronária percutânea; o aprimoramento terapêutico permitiu maiores índices de sucesso, com menor ocorrência de oclusão aguda, menor necessidade de cirurgia cardíaca de emergência e consequente menor mortalidade; e o acometimento coronário multiarterial, o infarto agudo do miocárdio e as lesões complexas estão associados a maior risco de cirurgia de emergência, enquanto a utilização de stent reduziu a chance dessa complicação.

Observou-se que, à medida que se avançou cronologicamente, houve aumento significativo tanto da idade dos pacientes como da maior participação porcentual do sexo feminino e de lesões complexas. Todas essas características são sabidamente relacionadas a complicações e pior prognóstico para intervenção coronária percutânea. ${ }^{2}$ Essa ampliação das indicações para revascularização por cateter também foi observada em estudos internacionais, como no National, Heart, Lung, and Blood Institute Registry (Registro NHLBI), no qual, com o passar do tempo, houve claro agravamento dos pacientes submetidos a intervenção coronária percutânea. ${ }^{3}$

Apesar da maior complexidade dos casos, houve melhora considerável das taxas de sucesso do procedimento. Esse fato pode ser explicado pela definição de sucesso angiográfico, que implica estenose residual menor que $20 \%$ intraprocedimento. Com o surgimento do stent, foi possível obter-se maior ganho luminal agudo no tratamento. De fato, estudos do período da angioplastia por balão demonstram índices de sucesso angiográfico entre $62 \%$ e $91 \%$, de acordo com a complexidade dos casos, com aumento gradual para patamares superiores a $90 \%$ a partir do surgimento dos stents. ${ }^{2,4-8}$

Grave complicação dos pacientes submetidos a intervenção coronária percutânea é a oclusão coronária aguda, que, na era do balão, ocorria em 4,3\% a 8,3\% 
TABELA 2

Estratégias terapêuticas no momento da intervenção percutânea nos períodos com disponibilidade de uso de stent

\begin{tabular}{lccc}
\hline & $\begin{array}{c}\text { Período } \\
\text { intermediário } \\
(\mathbf{n = 3 . 7 1 1 )}\end{array}$ & $\begin{array}{c}\text { Período } \\
\text { mais recente } \\
(\mathbf{n = 3 . 7 2 7 )}\end{array}$ & $\mathbf{P}$ \\
\hline AAS, \% & 98 & 98 & $>0,99$ \\
Clopidogrel, \% & 49,5 & 51 & 0,31 \\
IGP Ilb/IIla, \% & 25,7 & 26,3 & 0,70 \\
Betaboqueadores, \% & 63 & 68 & $<0,01$ \\
Estatinas, \% & 42,8 & 52,5 & $<0,01$ \\
IECA, \% & 45,2 & 49,3 & $<0,01$ \\
Utilização de stent/lesão, \% & 84,3 & 88,5 & $<0,01$ \\
\hline
\end{tabular}

AAS = ácido acetilsalicílico; IECA = inibidor da enzima conversora da angiotensina; IGP = inibidores da glicoproteína Ilb/IIla; $\mathrm{n}=$ número de pacientes.

TABELA 3

Preditores independentes da necessidade de cirurgia de emergência nos períodos com utilização de stents

\begin{tabular}{lcc}
\hline & OR & IC 95\% \\
\hline Padrão multiarterial & 3,42 & $1,75-6,7$ \\
Infarto agudo do miocárdio & 3,65 & $2,3-5,8$ \\
Lesão B2/C AHA/ACC & 3,27 & $1,6-6,75$ \\
Uso de stent & 0,58 & $0,4-0,85$ \\
\hline
\end{tabular}

$\mathrm{AHA} / \mathrm{ACC}=$ American Heart Association/American College of Cardiology; IC 95\% = intervalo de confiança de $95 \%$; $\mathrm{OR}=$ odds ratio.

das intervenções. ${ }^{11}$ Nesse aspecto, o acúmulo de conhecimento acerca da antiagregação plaquetária, com a utilização de tienopiridínicos e inibidores da glicoproteína Ilb/IIla, bem como o advento dos stents, reduziu significativamente essa complicação, ocorrendo na última fase em cerca de $1 \%$ dos casos. $^{12,13}$ Esses dados da literatura respaldam os achados de nosso registro, em que foi observada redução da ocorrência de oclusão aguda superior a sete vezes $(7,8 \%$ para o período da angioplastia convencional para 1,1\% no período mais recente).

Com maiores índices de sucesso do procedimento e redução da oclusão coronária aguda, houve progressiva diminuição da necessidade de cirurgia cardíaca de emergência. Em nossa população, mesmo nos períodos que envolvem o emprego do stent, a maior utilização dessa prótese pode ser considerada uma das responsáveis pela redução das cirurgias cardíacas. Esses dados são similares aos relatados por Seshadri et al., da Cleveland Clinic, que reportaram incidência de 0,61\%. ${ }^{9}$ Da mesma forma, o Registro da Mayo Clinic, com
23.087 pacientes, relata a necessidade de cirurgia de emergência de 2,9\% no período pré-stent, de $0,7 \%$ na era inicial do stent e de $0,3 \%$ na era atual do stent. ${ }^{10}$ Obtendo-se melhor tratamento clínico e percutâneo com menos complicações pode-se explicar a menor mortalidade observada com a modernização terapêutica.

Finalmente, os preditores de necessidade de cirurgia de emergência encontrados foram o padrão coronário multiarterial, a presença de infarto agudo do miocárdio e a complexidade das lesões (tipo B2/C). Dado que reforça a importância dos stents nesse quesito é o efeito protetor exercido por eles na necessidade da intervenção cirúrgica. Similarmente, Yang et al. ${ }^{10}$ identificaram infarto agudo do miocárdio, choque cardiogênico, lesões tipo C, acometimento triarterial e infarto agudo prévio como preditores independentes de cirurgia de revascularização miocárdica de emergência.

\section{Limitações do estudo}

Como limitações deste estudo podemos ressaltar o fato de a perspectiva histórica ter sido realizada em duas instituições distintas. No entanto, trata-se de hospitais-escola de grande volume e referências para o tratamento cardiológico de alta complexidade em suas épocas, constituindo o maior registro nacional nesse sentido. Também deve ser mencionado o fato de que os eventos documentados se restringem ao período intra-hospitalar. Esses resultados observados podem não se aplicar a centros de menor volume e não devem ser extrapolados.

\section{CONCLUSÃO}

Em conclusão, os resultados do presente estudo demonstram que os avanços da técnica, do material, uso dos stents e farmacoterapia adjunta têm contribuído com a intervenção coronária percutânea para ampliar suas indicações, com maior índice de sucesso e me- 
nor necessidade de cirurgia cardíaca de emergência e mortalidade.

\section{CONFLITO DE INTERESSES}

Os autores declararam inexistência de conflito de interesses relacionado a este manuscrito.

\section{REFERÊNCIAS}

1. Gruntzig A. Transluminal dilatation of coronary-artery stenosis. Lancet. 1978;1:263.

2. Smith SC Jr, Feldman TE, Hirshfeld JW Jr, Jacobs AK, Kern MJ, King SB $3^{\text {rd }}$, et al. ACC/AHA/SCAl 2005 guideline update for percutaneous coronary intervention: a report of the American College of Cardiology/American Heart Association Task Force on Practice Guidelines (ACC/AHA/SCAI Writing Committee to Update 2001 Guidelines for Percutaneous Coronary Intervention). Circulation. 2006;113(7):e166-286.

3. Williams DO, Holubkov R, Yeh W, Bourassa MG, Al-Bassam $\mathrm{M}$, Block PC, et al. Percutaneous coronary intervention in the current era compared with 1985-1986: the National Heart, Lung, and Blood Institute Registries. Circulation. 2000;102(24): 2945-51.

4. Ellis SG, Vandormael MG, Cowley MJ, DiSciascio G, Deligonul $\mathrm{U}$, Topol EJ, et al. Coronary morphologic and clinical determinants of procedural outcome with angioplasty for multivessel coronary disease: implications for patient selection. Multivessel Angioplasty Prognosis Study Group. Circulation. 1990;82(4): 1193-202.

5. King SB, Yeh W, Holubkov R, Baim DS, Sopko G, DesvigneNickens $P$, et al. Balloon angioplasty versus new device intervention: clinical outcomes. A comparison of the NHLBI PTCA and NACl registries. J Am Coll Cardiol. 1998;31(3): 558-66.

6. Feit F, Brooks MM, Sopko G, Keller NM, Rosen A, Krone R, et al. Long-term clinical outcome in the Bypass Angioplasty
Revascularization Investigation Registry: comparison with the randomized trial. BARI Investigators. Circulation. 2000; 101(24):2795-802.

7. McGrath PD, Malenka DJ, Wennberg DE, Shubrooks SJ Jr, Bradley WA, Robb JF, et al. Changing outcomes in percutaneous coronary interventions: a study of 34,752 procedures in northern New England, 1990 to 1997. Northern New England Cardiovascular Disease Study Group. J Am Coll Cardiol. 1999;34(3):674-80.

8. Srinivas VS, Brooks MM, Detre KM, King SB $3^{\text {rd }}$, Jacobs AK, Johnston J, et al. Contemporary percutaneous coronary intervention versus balloon angioplasty for multivessel coronary artery disease: a comparison of the National Heart, Lung and Blood Institute Dynamic Registry and the Bypass Angioplasty Revascularization Investigation (BARI) study. Circulation. 2002; 106(13):1627-33.

9. Seshadri N, Whitlow PL, Acharya N, Houghtaling P, Blackstone $\mathrm{EH}$, Ellis SG. Emergency coronary artery bypass surgery in the contemporary percutaneous coronary intervention era. Circulation. 2002;106:2346-50.

10. Yang EH, Gumina RJ, Lennon RJ, Holmes DR Jr, Rihal CS, Singh M. Emergency coronary artery bypass surgery for percutaneous coronary interventions: changes in the incidence, clinical characteristics and indications from 1979 to 2003. J Am Coll Cardiol. 2005;46(11):2004-9.

11. de Feyter PJ, de Jaegere PP, Serruys PW. Incidence, predictors, and management of acute coronary occlusion after coronary angioplasty. Am Heart J. 1994;127(3):643-51.

12. Cutlip DE, Baim DS, Ho KK, Popma JJ, Lansky AJ, Cohen DJ, et al. Stent thrombosis in the modern era: a pooled analysis of multicenter coronary stent clinical trials. Circulation. 2001;103(15):1967-71.

13. Ong AT, Hoye A, Aoki J, van Mieghem CA, Rodriguez Granillo GA, Sonnenschein K, et al. Thirty-day incidence and six month clinical outcome of thrombotic stent occlusion after baremetal, sirolimus, or paclitaxel stent implantation. J Am Coll Cardiol. 2005;45(6):947-53. 